\title{
Problemas de programação geométrica signomial via otimização DC
}

\author{
Clarissa Pessoa Borges Fernandes \\ Faculdade Maurício de Nassau \\ João Pessoa, PB \\ E-mail: borgesclarissa@gmail.com \\ Roberto Quirino do Nascimento \\ UFPB - Centro de Informática \\ Campus I \\ 58051-900, João Pessoa, PB \\ E-mail: quirino@ci.ufpb.br \\ Ana Flávia Uzeda dos Santos Macambira \\ UFPB - Departamento de Estatística \\ Campus I \\ 58051-900, João Pessoa, PB \\ E-mail: af.macambira@gmail.com
}

\begin{abstract}
Resumo: Neste trabalho os Problemas de Programação Geométrica Signomial, não convexos, são escritos em sua forma posinomial e posteriormente reescritos na forma padrão (CDC) de um problema de diferença de funções convexas $(D C)$, definidos por $D C G$ (DC geométrico). Tal metodologia foi utilizada pois permite que se obtenha a solução global para o problema. Com base na técnica de otimização global, Otimização DC, foi proposto um algoritmo e foram resolvidos problemas clássicos da literatura de Programação Geométrica onde os resultados obtidos foram iguais e em alguns casos melhores do que os já existentes. Esta melhora na solução se deve ao fato de que, em geral, os resultados encontrados na literatura são obtidos através da implementação de metaheuristicas.
\end{abstract}

Palavras-chave: Programação Geométrica Signomial; Otimização DC; Otimização Global.

Neste trabalho é feita uma abordagem da teoria de Problemas de Programação Geométrica e da teoria de Otimização de Diferença de Funções Convexas(DC) onde mostra-se que uma classe dos problemas de Programação Geométrica, conhecida por Problemas Signomiais, pode ser escrita como a diferença de funções convexas e mais adiante, que um problema DC pode ser escrito na forma CDC que é a forma canônica do problema DC. A vantagem de se escrever na forma CDC é que pode-se encontrar a solução global para este tipo de problema. Os problemas resolvidos tem como referência os artigos Nenad [5], Maranas [4] e Dembo [2].

Os Problemas de Programação Geométrica podem ser Posinomiais ou Signomias. Os problemas posinomiais são caracterizados por funções de termos positivos enquanto que os signomiais possuem pelo menos um termo negativo. Soluções para problemas posinomiais podem ser encontradas através de algoritmos tais como, Branch and Bound e Método de Pontos Interiores. No caso dos problemas signomiais, existem algumas particularidades, entre elas a não convexidade da função objetivo que torna mais difícil a sua resolução.

Problemas de Programação Geométrica Generalizada (PGG), também chamada de Programação Geométrica Signomial (PGS), são caracterizados por funções objetivos e restrições descritas como a diferença de dois posinômios. Os PGSs contém um ou mais monômios com coeficientes negativos [4]. Para esta classe de problemas em muitos casos é possível determinar 
um mínimo local e para encontrar a solução global é necessário utilizar técnicas de otimização global. Uma dessas técnicas para resolver tais problemas é transformar a função objetivo na diferença de duas funções convexas.

Os problemas de Otimização DC, onde a região viável é um conjunto convexo e a função objetivo é a diferença entre duas funções convexas, aqui chamado de "problema geral de otimização DC". Problemas de Otimização DC tem aplicações nas áreas de economia, engenharia e outros, como podem ser visto nos artigos Tuy [6], Tuy [7] e Hoai [3]. Este tipo de problema cobre os problemas de otimização convexa padrão e minimização côncava. De fato, qualquer problema de Otimização contínuo sobre um conjunto compacto pode ser transformado como um problema de Otimização DC [6]. Todos os problemas de otimização DC podem ser reduzidos a forma de Problemas Canônicos de Otimização DC (CDC), que tem uma estrutura matemática mais simples que a do problema geral de otimização DC. Assim, algoritmos para resolver o problema CDC possibilitam um tratamento único aos problemas de otimização DC.

Programação Geométrica é uma técnica de otimização utilizada para resolver algebricamente problemas de otimização não-linear. Foi desenvolvida no início da década de 60 por Clarence Melvin Zener (1905 - 1993), Richard J. Duffin (1909 - 1996) e Elmor L. Peterson(1938). Em 1961 Zener descobriu uma maneira simples de minimizar funções da forma:

$$
g_{k}(t)=\sum_{i \in J[k]} c_{i} \prod_{j=1}^{m} t_{j}^{a_{i j}} \quad k=0,1, \ldots, p
$$

Num problema de Programação Geométrica as parcelas existentes na função objetivo e nas restrições são chamadas de posinômios e a função objetivo é chamada função posinomial. É denominada desta forma devido ao relacionamento existente entre as médias geométricas e aritméticas. Problemas caracterizados por funções objetivos e/ou restrições que envolvem a diferença de dois ou mais posinômios são chamados de Problemas de Programação Signomial. Para esta classe de problemas em muitos casos sé é possível determinar um mínimo local. Para determinar a solução global é necessário utilizar técnicas de otimização global. Um Problema de Programação Geométrica é da forma:

$$
\min g_{0}(t)
$$

Sujeito a:

$$
g_{k}(t) \leq 1, k=1, \ldots, p, t>0
$$

onde

$$
\begin{gathered}
g_{k}(t)=\sum_{i \in J[k]} \sigma_{i} c_{i} \prod_{j=1}^{m} t_{j}^{a_{i j}} \quad k=0,1, \ldots, p \\
J[k]=\left\{m_{k}, m_{k+1}, \ldots, n_{k}\right\} \quad k=0,1, \ldots, p \\
m_{0}=1, \quad m_{1}=n_{0}+1, m_{2}=n_{1}+1, \ldots, m_{p}=n_{p-1}+1, \quad n_{p}=n
\end{gathered}
$$

as constantes $c_{i}$ são positivas, $a_{i j}$ são números reais. Quando $\sigma_{i}=1$ o problema é dito posinomial e quando existir pelo menos uma ocorrência de $\sigma_{i}=-1$, o problema é dito signomial.

Devido a existência de termos negativos, o problema signomial não pode ser convertido para um problema convexo usando transformações do tipo $t_{j}=e^{z_{j}}, z_{j} \in \Re, \quad j=1, \cdots, m$. Desta forma os Problemas Programação Geométrica Signomiais (PGS) são problemas não convexos e qualquer ponto que satisfaça as condições de Karush-Kuhn-Tucker pode ser apenas um mínimo local ou apenas um ponto de sela. Tal fato levou inicialmente os pesquisadores a tentarem resolver este tipo de problema usando programação geométrica sequencial, que consiste em resolver problemas signomiais através de uma sequência de problemas de programação geométrica posinomial. Esta metodologia denomina-se condensação. 
Muitos problemas de otimização envolvem funções que podem ser expressadas como a $d i$ ferença entre funções convexas (funções DC). Um resultado importante é que toda função de classe C-2 tem uma representação DC, veja TUY [8].

Uma função $f$ definida em um conjunto convexo $X \subseteq \Re^{n}$ é dita DC em $X$ se, para todo $x \in X, f$ pode ser expressada como:

$$
f(x)=p(x)-q(x)
$$

onde $p, q$ são funções convexas em $X$.

Uma função que é uma diferença de funções convexas em $\Re^{n}$ é chamada de função DC. A representação 4 é uma decomposição de $f$ como uma função DC.

Problema de otimização global é chamado de um problema de programação DC se ele pode ser escrito da forma:

$$
\begin{aligned}
\text { Minimizar } & f_{0}(x) \\
\text { s.a. } & x \in X, \\
& f_{i}(x) \leq 0,(i=1, \ldots, m)
\end{aligned}
$$

Uma característica interessante da programação DC é que alguns problemas que podem ser escritos como o que aparece em 5 podem ser reduzidos ao problema canônico de minimizar uma função linear sobre a intersecção de um conjunto convexo com o complementar de um conjunto convexo aberto. O complementar de um conjunto convexo aberto é usualmente descrito como uma restrição convexa reversa, e tem a forma:

$$
g(x) \geq 0
$$

onde $g: \Re^{n} \rightarrow \Re$ é uma função convexa. Um problema canônico DC é um problema de otimização da forma

$$
\begin{aligned}
\text { Minimizar } & c^{T} x \\
\text { s.a. } & x \in D \\
& g(x) \geq 0
\end{aligned}
$$

onde $c \in \Re^{n}, g: \Re^{n} \rightarrow \Re$ é convexa, e $D$ é um subconjunto convexo compacto do $\Re^{n}$.

Seja $f, f_{i}(i=1, \ldots, m)$ funções DC. Então as seguintes funções também são DC.

1. $\sum_{i=1}^{m} \lambda_{i} f_{i}(x)$ para algum número real $\lambda_{i}$;

2. $\max _{i=1, \ldots, m} f_{i}(x)$ e $\min _{i=1, \ldots, m} f_{i}(x)$;

3. $|f(x)|, f^{+}:=\max \{0, f(x)\}, f^{-}:=\min \{0, f(x)\}$;

4. o produto $\prod_{i=1}^{m} f_{i}(x)$.

Condições de Otimalidade para o problema de otimização DC:

Considere um par de problemas de programação DC dados por:

$$
\omega^{*}{ }_{\delta}=\inf \{\omega(z): z \in Z, \psi(z) \leq \delta\}
$$

e

$$
\psi^{*}{ }_{\eta}=\inf \{\psi(z): z \in Z, \omega(z) \leq \eta\}
$$

onde $Z \subseteq \Re^{p}, \delta, \eta \in \Re$ e $\omega$ e $\psi$ são funções finitas em $\Re^{p}$. Seja $\Omega^{*}{ }_{\delta}$ e $\Psi^{*}{ }_{\eta}$ o conjunto das soluções ótimas dos Problemas 8 e 9, respectivamene. Seja $Z \subset \Re^{p}, f: \Re^{p} \rightarrow \Re$, e $\delta \in \Re$. O conjunto

$$
S(Z, f, \delta)=\{z \in Z: f(z) \leq \delta\}
$$


é dito robusto se

$$
S(Z, f, \delta)=\operatorname{cl}(\{z \in Z: f(z)<\delta\})
$$

onde para cada conjunto $S, c l(S)$ é o fecho de $S$. Se nos problemas 8 e 9 a função $\omega$ é convexa e a condição 10 é satisfeita

$$
\exists z^{0} \in Z: \omega\left(z^{0}\right)<\omega^{*}
$$

, então o conjunto $S(Z, \omega, \eta)$ é robusto para cada $\eta \geq \omega^{*}$.

Considerando um caso especial do problema 8

$$
\omega^{*}{ }_{\delta}=\inf \{\omega(z): z \in Z, \psi(z) \leq 0\} .
$$

Assuma que no problema 11 a função $\omega$ é convexa, o conjunto $S(Z, \psi, 0)=\{z \in Z: \psi(z) \leq 0\}$ é robusto e a condição 10 é satisfeita. Então o ponto viável $z^{*}$ de 11 é uma solução ótima se e somente se

$$
0=\inf \left\{\psi(z): z \in Z, \omega(z) \leq \omega\left(z^{*}\right)\right\} .
$$

Uma importante classe de problemas de otimização DC é:

$$
\omega^{*}=\inf \{g(x)-h(x): x \in X\}
$$

onde $g$ e $h$ são duas funções convexas em $\Re^{n}$ e $X$ é um subconjunto fechado convexo do $\Re^{n}$. $\mathrm{O}$ resultado seguinte fornece uma condição de otimalidade para o problema 13. Assuma que o problema 13 tem solução. Então o ponto $x^{*} \in X$ é uma solução ótima se e somente se existe $t^{*} \in \Re$ tal que

$$
0=\inf \left\{-h(x)+t: x \in X, t \in \Re, g(x)-t \leq g\left(x^{*}\right)-t^{*}\right\} .
$$

Todo problema de Otimização DC é equivalente a um problema na forma CDC em $\Re^{n+2}$. Considere o problema canônico DC (CDC) com o conjunto viável $F=\{x: g(x) \geq 0\}$, exige-se que o problema CDC satisfaça algumas das seguintes hipóteses:

- $\left(H_{1}\right) D$ é compacto e int $D \neq \emptyset$.

- $\left(H_{2}\right)$ existe um ponto $x^{0} \in D$ satisfazendo $g\left(x^{0}\right)<0$ e $c^{T} x^{0}<\min \left\{c^{T} x: x \in D, g(x) \geq 0\right\}$.

- $\left(H_{3}\right) F=\operatorname{cl}($ int $F)$.

A suposição $\left(H_{2}\right)$ significa que $g(x) \geq 0$ é essencial, pois se $\left(H_{2}\right)$ não é satisfeita então o problema (CDC) é equivalente ao problema de minimização convexa:

$$
\begin{aligned}
\text { Minimizar } & c^{T} x \\
\text { s.a. } & x \in D
\end{aligned}
$$

A terceira propriedade, é a robustez do conjunto viável $F=\{x \in D: g(x) \geq 0\}$ de (CDC). Isto significa, em particular, que é excluído o caso onde a intersecção dos conjuntos $\{x: g(x) \geq 0\}$ e $D$ é uma parte da fronteira de $D$.

No problema CDC, assuma que $D$ é limitado, $F$ é não vazio e a restrição convexa reversa $g(x) \geq 0$ é essencial. Então existe uma solução ótima na intersecção $\partial D \cap \partial G$ que são as fronteiras de $D$ e $G$.

(Condição de otimalidade necessária) No problema CDC, sejam as hipóteses $H_{1}$ e $H_{2}$ satisfeitas. Então toda solução ótima $\bar{x}$ de CDC satisfaz

$$
\max \left\{g(x): x \in D, c^{T} x \leq c^{T} \bar{x}\right\}=0
$$


(Condição de otimalidade suficiente) Assuma que no problema CDC o conjunto viável $F$ é robusto (condição $H_{3}$ é satisfeita) e que o ponto $x^{0}$ satisfazendo $g\left(x^{0}\right)<0$ existe. Seja $\bar{x} \in F$ e $S \supseteq F$ tal que

$$
\max \left\{g(x): x \in S, c^{T} x \leq c^{T} \bar{x}\right\}=0 .
$$

Então $\bar{x}$ é uma solução ótima de CDC.

O problema (PGS) pode ser escrito como a diferença de funções convexas e tem a seguinte forma:

$$
\begin{aligned}
\text { Minimizar } & G_{0}(z)=G_{0}^{+}(z)-G_{0}^{-}(z) \\
\text { s.a. } & G_{k}(z)=G_{k}^{+}(z)-G_{k}^{-}(z) \leq 0 \quad k=1,2, \cdots, p \\
& z_{j}^{L} \leq z_{j} \leq z_{j}^{U} \quad j=1,2, \cdots, m
\end{aligned}
$$

Sendo,

$$
G_{k}^{+}(z)=\sum_{i \in J^{+}[k]} c_{i} e^{\sum_{j=1}^{m} a_{i j} z_{j}} G_{k}^{-}(z)=\sum_{i \in J^{-}[k]} c_{i} e^{\sum_{j=1}^{m} a_{i j} z_{j}}, \quad k=1,2, \cdots, p
$$

Para que $z_{j}^{L}=\ln \left(x_{j}^{L}\right)$, é necessário que $t_{j}^{L}>0$, para $j=1,2, \cdots, m$.

Uma propriedade importante da programação geométrica é que quando o problema primal tem a forma posinomial, este admite uma reformulação convexa. Em Yang [9] discute-se como resolver um problema signomial aproximando-o por um problema posinomial. De acordo com Beightler [1] dois casos podem ocorrer em um problema de PG signomial: a função objetivo ser signomial ou ser posinomial.

Caso 1: A função objetivo é posinomial, ou seja, $J^{-}[k]=\emptyset$.

Neste caso é criada uma variável $t_{0}$ e a sua forma CDC fica como segue. Ou seja, um problema com função objetivo linear onde as restrições podem ser posinimiais ou signomiais.

$$
\begin{aligned}
\text { Minimizar } & t_{0} \\
\text { s.a. } & H_{1}(t) z^{-1} \leq 1, \\
& \left(1+H_{2}(t)\right) z^{-1} \geq 1 \\
& t, t_{0} \in D \\
& z>0
\end{aligned}
$$

onde

$G(t)=\operatorname{Max}\left\{g_{k^{+}}(t)-g_{k^{-}}(t)\right\}=\operatorname{Max}\left\{g_{k^{+}}(t)+\sum_{l=0, l \neq k}^{p} g_{k^{-}}(t)\right\}-\sum_{k=0}^{p} g_{k^{-}}(t)=H_{1}(t)-H_{2}(t)$,

$D$ é definido por

$$
D=\left\{\begin{array}{l}
\left(t, t_{0}\right) ; \quad g_{k}(t) t_{0}^{-1} \leq 1 \\
g_{k}(t) \leq 1 \text { se } \quad J^{-}[k]=\emptyset \\
L \leq t_{i} \leq U, \quad i=1, \ldots, m
\end{array}\right.
$$

e $H_{1}$ e $H_{2}$ são funções posinomiais.

Caso 2: A função objetivo é signomial.

Minimizar $g_{0}(t)-h_{0}(t)$ é equivalente a:

$$
\begin{aligned}
\text { Minimizar } & t_{0} \\
\text { s.a. } & g_{0}(t)-h_{0}(t) \leq f\left(t_{0}\right) \\
& t, t_{0} \geq 0
\end{aligned}
$$


Onde $f: \Re_{+} \longrightarrow \Re$ definida por $f\left(t_{0}\right)=\sqrt{t_{0}}-\frac{1}{\sqrt{t_{0}}}$. Escrevendo o Problema de Programação Geométrica Signomial (PGS) na forma padrão DC, na qual será chamado forma padrão DC Geoométrica.

$$
\begin{aligned}
\text { Minimizar } & t_{0} \\
\text { s.a. } & h\left(t, t_{0}\right) t_{m+2}^{-1} \geq 1 \\
& g_{k}^{-}\left(t, t_{0}\right) t_{m+1}^{-1} \leq 1, k=0, \cdots, p \\
& t_{m+1} t_{m+2}^{-1} \leq 1 \\
& t_{m+1}>0 \\
& t_{m+2}>0 \\
& l_{1} \leq t_{i} \leq U_{i}, \quad i=1, \ldots, m
\end{aligned}
$$

Onde:

$$
\begin{aligned}
& h\left(t, t_{0}\right)=\left\{\begin{array}{lll}
h_{0}(t) t_{0}^{-\frac{1}{2}}+\sum_{k=1}^{p} \sum_{i \in J^{-}[k]} c_{i} \prod_{j=1}^{m} t_{j}^{a_{i j}}, & \text { se } \quad J^{-}[0]=\emptyset \text { e } J^{-}[k] \neq \emptyset \\
\sum_{k=1}^{p} \sum_{i \in J^{-}[k]} c_{i} \prod_{j=1}^{m} t_{j}^{a_{i j}}, & \text { se } \quad J^{-}[k] \neq \emptyset
\end{array}\right.
\end{aligned}
$$

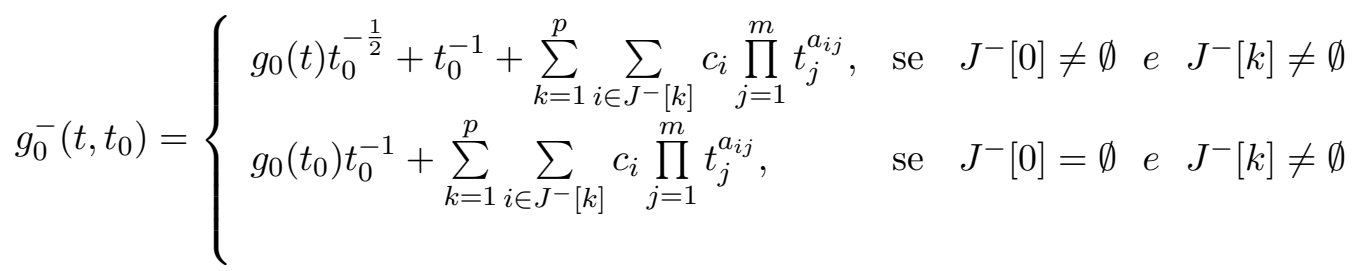

$$
\begin{aligned}
& g_{k}^{-}(t)=\left\{\begin{array}{lll}
g_{k}(t)+\sum_{l=1 ; l \neq k}^{p} \sum_{i \in J^{-}[k]} c_{i} \prod_{j=1}^{m} t_{j}^{a_{i j}}, & \text { se } & J^{-}[k] \neq \emptyset \\
g_{k}(t), & \text { se } & J^{-}[k]=\emptyset
\end{array}\right.
\end{aligned}
$$

Neste trabalho, o problema de programação geométrica signomial foi escrito na forma padrão do problema de diferença de funções convexas, pois foi mostrado que, mesmo a função objetivo sendo signomial o problema pode ser reescrito com uma função objetivo posinomial. Feito isto, tem-se um Problema de Programação Geométrica Signomial com função objetivo posinomial (com um único termo) e com restrições signomiais que estas são escritas como funções DC. Ao reescrever o Problema PGS na forma CDC, ou seja na forma DC Geométrica, obtém-se um problema equivalente ao original e cuja solução, quando encontrada, é global.

Na tabela abaixo estão os resultados para comparação das soluções encontradas nos problemas DC geométrico e das soluções encontradas na literatura. 


\begin{tabular}{|c|l|l|l|l|}
\hline Problema & $\begin{array}{l}\text { Solução Problema DC } \\
\text { Geométrico }\end{array}$ & $\begin{array}{l}\text { Solução DEMBO, } \\
\text { R.S. }\end{array}$ & $\begin{array}{l}\text { Solução N. Mla- } \\
\text { denovic et al. }\end{array}$ & $\begin{array}{l}\text { Solução } \\
\text { Rijckaert- } \\
\text { Martens }\end{array}$ \\
\hline \hline 1 & 11.964354188901051 & & & 11.96 \\
\hline 2 & -83.166292876248477 & & -83.2535 & -83.21 \\
\hline 3 & -5.739820299189251 & & -5.7398 & -5.7398 \\
\hline 4 & -6.048232613753129 & & -6.0482 & -6.0482 \\
\hline 5 & 7049.259701907435 & 7049.324305 & 7049.25 & 7049.247 \\
\hline 6 & 1.143623168507892 & & 1.1437 & 1.1436 \\
\hline 7 & 0.205653557690020 & & & 0.2015 \\
\hline 8 & 0.140611630536061 & & 0.1406 & 0.1406 \\
\hline 9 & 10122.50013983291 & 10126.64252 & 10122.6964 & \\
\hline 10 & 1213.109972529761 & 1227.1831610 & 1227.23 & \\
\hline 11 & 3.951248832495454 & 3.9516982 & 3.9511 & \\
\hline 12 & 97.599994628275937 & 97.591034 & & \\
\hline
\end{tabular}

\section{Referências}

[1] C. S. Beightler, D. T. Phillps, Applied Geometric Programming. (John Wiley Sons) United States, 1976.

[2] R.S. Dembo, , (1976), A Set of Geometric Programming test problems and their Solutions, Mathematical Programming 10, 192-213.

[3] L.T. Hoai An, ; P.D.Tao, A continuous approach for globally solving linearly constrained quadratic zero-one programming problems , Optimization, 50, 93120, 2001.

[4] C.D. Maranas , C.A. Floudas, Global Optimization in Generalized Geometric Programming, Computers chem. Engng Vol. 21, No. 4, pp. 351-369, 1997 Copyright,1996 - Elsevier Science Ltda.

[5] N. Mladenovic, et al. General Variable Neighborhood Search for the Continuous Optimization, European Journal of Operational Research 191 (2008) 753770.

[6] H.Tuy; ,F.Al-Khayyal; F.Zhou; A DC Optimization Method for Single Facility Location Problems J. Global Optim., 7: 209-227, 1995.

[7] H.Tuy; Convex Analysis and Global Optimization, Honai, Vietnam: Kluwer Academic Publishers, 1998.

[8] H.Tuy; Canonical DC programming problem: Outer approximation methods revisited, Operations Research Letters 18, 99-106, 1995.

[9] H.Yang, D. L. Bricker. Investigation of Path-Following Algoritms for Signomial Geometric Programming Problems. European Journal of Operational Research, 103:230241.(1996) 